\title{
Hydraulically Powered Wood Chip Blower in Test and Its Use Opportunity in Biofuel Supply Chain
}

\author{
Jarno Föhr1*, Raghu KC1, Tapio Ranta1, Markku Miettinen² \\ ${ }^{1}$ Laboratory of Bioenergy, Lappeenranta-Lahti University of Technology LUT, Mikkeli, Finland \\ ${ }^{2}$ Lämpösi Oy, Jyväskylä, Finland \\ Email: *jarno.fohr@lut.fi
}

How to cite this paper: Föhr, J., KC, R., Ranta, T. and Miettinen, M. (2020) Hydraulically Powered Wood Chip Blower in Test and Its Use Opportunity in Biofuel Supply Chain. Journal of Sustainable Bioenergy Systems, 10, 77-91.

https://doi.org/10.4236/jsbs.2020.103007

Received: June 8, 2020

Accepted: August 28, 2020

Published: August 31, 2020

Copyright $\odot 2020$ by author(s) and Scientific Research Publishing Inc. This work is licensed under the Creative Commons Attribution International License (CC BY 4.0).

http://creativecommons.org/licenses/by/4.0/

\begin{abstract}
Most forest chips are delivered to large-sized, combined heat and power plants in Finland. In this study, we introduce and demonstrate the small-scale delivery of forest wood chips to potential clients, with the wood chips being delivered in a container truck and pneumatically blown into a storage facility. This arrangement of wood chips being blown through a pipe into a storage eliminates the ground handling while requiring a relatively small space. In the demonstration tests, we tested the volumetric flow of biomass in the hose and its noise level while the blower was blowing the material and the flow speed of the material in the hose. This study discusses the test conditions in which the compatibility and suitability of the truck and its hydraulic system were used in conventional chip delivery in Finland and the selected blower type was investigated. According to the results, the biomass volume flow was higher $\left(\sim 0.46 \mathrm{~m}^{3} / \mathrm{min}\right)$ when the blower revolution was lower (2392 - $\left.2566 \mathrm{RPM}\right)$. However, when the RPM was increased to $\sim 3000$, the volumetric flow decreased to $\sim 0.24 \mathrm{~m}^{3} / \mathrm{min}$. Similarly, the speed of the chips was higher with a lower RPM; $29.9 \mathrm{~m} / \mathrm{s}$ at an RPM of 2400 and $25 \mathrm{~m} / \mathrm{s}$ at an RPM of 2700 . This is potentially due to both the blower and screw conveyer using the same power source. Additional optimization research would be needed to conclusively state the root cause of this phenomenon. On the other hand, chips from pruned stems had better flow than the chips from whole trees, since chips from whole tree have a wider range of chips sizes, including $1.3 \%$ of chips being in the range of $31.5-40 \mathrm{~mm}$. The larger chips clogged the hose, which hindered the flow. Finally, the noise tests showed that it was louder at 0 degrees, which contrasted with the situation for the blower, with an aside at 90 degrees.
\end{abstract}


Keywords

Truck, Blower, System, Test, Biomass, Supply Chain

\section{Introduction}

Biomass is considered as an important renewable alternative to fossil fuels in energy generation [1]. Many countries, including Finland, are aiming to eliminate coal from energy production and biomass is the foremost candidate to replace it [2]. On the other hand, biomass is abundantly available in Finland and wood fuel alone contributes $27 \%$ of the consumed energy in Finland. It is important to note that a significant share of biomass energy goes into the heating sector [3]. Furthermore, a significant part of such energy comes from large-scale combined heat and power (CHP) power plants as a district heating network [4]. However, in rural Finland, where such a district heating network is not available, households must turn to stand-alone heating systems, such as chips/pellets fired heating, direct electricity heating, heat pumps, and oil-fired heating. According to the statistics on heating systems, direct electricity (34\%), district heating (28\%), wood (22\%), ambient energy (9\%), and light fuel oil (5\%) constitute the major sources of energy for heating [5].

In the case of using biomass for a heating system in a decentralized set up, biomass poses additional challenges compared to its competitors, such as handling and storage issues. In addition, the moisture content of biomass is a sensitive issue because it affects the efficiency of the whole heating system, thus requiring dry space for storage. In addition, most forest chips are delivered to CHP plants on trucks and trailers, and the small-scale delivery of forest chips is not very common in Finland. For these reasons, many households are turning away from biomass-powered heating [6]. The advantages and disadvantages of traditional and small-scale delivery are compared in Table 1 . This research paper investigates the practicalities of delivering forest chip on a small-scale to heating consumers.

Table 1. The advantages and disadvantages of traditional and small-scale delivery.

\begin{tabular}{lll}
\hline & Advantage & Disadvantage \\
\hline & + Larger volume capacity & \\
Traditional delivery & + Fewer transport quantities & - Large loading/unloading space \\
(full-trailer truck) & + Lower transport costs & - Slower loading/unloading \\
& + Suitable for long distances & \\
& & \\
Small-scale delivery & + Small loading/unloading space & - Several transport quantities \\
(truck/lorry) & + Faster loading/unloading & - Higher transport costs \\
& & - Unsuitable for long distances
\end{tabular}


With this new delivery solution, wood chips are blown pneumatically into a fuel storage system. Wood chip trucks can be unloaded by using a wood chip blower and a hose into desired space or tall silos, like an oil truck. This new blowing method eliminates the ground handling while requiring a relatively small space for unloading. The unloading method does not get dirt or mess the places, because the chips go through the hose directly to the fuel storage. With these improvements, pneumatic blowing can provide the necessary solution in favor for small-scale delivery. The unloading technique of the new delivery solution is also suitable for wood pellets and other solid biomass, such as straw, bark, and other similar materials.

The main target groups of the blowing system are the solid biofuel manufacturers and suppliers as well as the logistics carriers and the manufacturers of trucks superstructures. In addition, the secondary target groups are the boiler manufacturers, energy producers, and energy users with an energy production solution that requires an upgrade or a change. The latter target groups are related to the entire heating chain for wood chips.

The main objective was to test and measure the pneumatic transfer of wood chips for cases in which the unloading takes place directly from the truck's container to a fuel storage facility. Measurements included the quality of the chips, the rotation speed of the turbine, the volume flow of the chips, chip speed, and the noise level of the blower. The secondary objective was to introduce the technology behind the blowing concept and the supply chain alternatives for wood chip delivery. The study promotes wood-chip heating at large properties and small heating plants, especially when replacing older oil-fired boilers with biomass or wood chip boilers.

\section{Technology behind the Blowing Concept and Supply Chain Options}

\subsection{Blowing System}

This new pneumatic blowing system can also be used for applications other than just biomass supply logistics. Such applications may include landscaping and gardening, where biomass can be blown into planting areas for weed control. Moreover, litter and feed can be blown onto farms and into horse stables. In addition, sawdust can be blown onto jogging trails in outdoor areas and snow storages can be filled in ski resorts for the winter. Blowers can also be used to spread mineral wool at construction sites [7].

The French company Romanet Sarl patented this solution for a solid biomass delivery system based on a wood chip blower. In 2007, the first wood chip blower was developed by a home smithy of the company [8]. Utilizing this renewed technology, the Finnish company Lämpösi Oy has adapted the new solution to fit the situation in Finland, and they have licensed it to themselves [7]. Therefore, chip blowers have also been manufactured in Finland since the beginning of the 2020s. 
Currently, the blower models come in four sizes, depending on the diameter of the blades: JYJ400, JYJ500, JYJ600, and JYJ700. The blowing capacity varies depending on the material and the blower model (Table 2). The blowing capacity is $0.3-1.2 \mathrm{bulk}-\mathrm{m}^{3} / \mathrm{min}$ with wood chips and $5-15 \mathrm{t} / \mathrm{h}$ with wood pellets [8].

The driving force is obtained from the electric motor for the smaller blowers, such as with models JYJ400 and JYJ500. Alternatively, the blower can operate independently as an internal combustion engine (Figure 1). The smaller blowers are normally mobile and are suitable for spreading biomass for landscaping and farming purposes. Usually, the spreadable materials are litter, feed, and fertilizer. [7] The larger blowers, such as the JYJ600 and JYJ700, are powered by the vehicle's hydraulic system [7]. The blower can also be installed between two walls at the rear of the load space. The latter wall protects the blower against damage and reduces the noise of the turbine. Model JYJ600 was available for this paper's pneumatic blowing tests in 2019-2020 (Figure 2).

A slightly different blower is used to blow wood pellets, one which does not break the pellet's surface when blowing them. Pellets are normally transported in large, flexible bulk bags $\left(1 \mathrm{~m}^{3}\right)$, so the new blowing technology makes it possible to remove the crane from the vehicle. All blowers can also be used as the exhauster in the other direction [7].



Figure 1. The mobile blower JYJ500 [7].

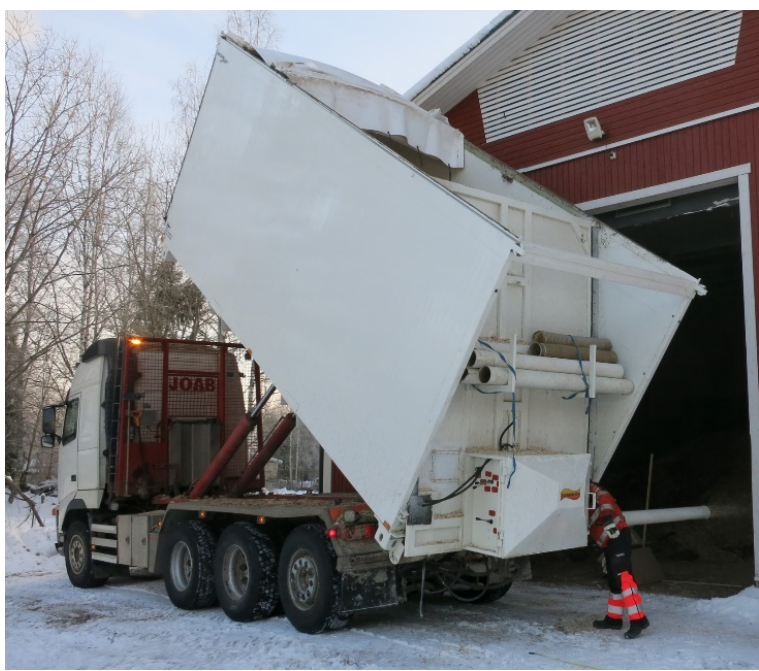

Figure 2. Model JYJ600 installed in a transfer container for blowing tests. 
Table 2. Capacity information for JYJ400-JYJ700 models [8].

\begin{tabular}{cccc}
\hline & Blowing distance $(\mathrm{m})$ & Capacity, wood chips $\left(\right.$ bulk- $\left.\mathrm{m}^{3} / \mathrm{min}\right)$ & Capacity, pellet $(\mathrm{t} / \mathrm{h})$ \\
\hline JYJ400 & $>15$ & $0.3-0.6$ & $5-6$ \\
JYJ500 & $>20$ & $0.5-0.7$ & $8-10$ \\
JYJ600 & $<40$ & $0.6-1.0$ & $8-12$ \\
JYJ700 & $<40$ & $0.8-1.2$ & $10-15$ \\
\hline
\end{tabular}

\subsection{Synergy between Container and Blower}

A new KOME composite transfer container (plastic composite) was used in the tests, where the wood chips could slide on the container's surface due to the slippery and non-freezing nature of the surface (Figure 2) [9]. Freezing problems have typically occurred especially when metal containers are used. As a result, the chips usually freeze onto the surface of the metal container, preventing the slipping of the chips. The container itself was a normal transfer container commonly used for the delivery of wood chips in Finland. The internal dimensions of the container were as follows: length $6 \mathrm{~m}$, height $2.7 \mathrm{~m}$, and width $2.4 \mathrm{~m}$. Total volume of container was $36.6 \mathrm{~m}^{3}$. The volume of the container would have been larger, but the fact that the rear wall was at an angle of 30 degrees in relation to the vertical wall reduced the volume by $2.3 \mathrm{~m}^{3}$ from the theoretical box model.

The maximum tip angle of the truck was 49 degrees. Figure 3 shows a schematic diagram of the chip blower connected to the transfer container. The main principle of pneumatic blowing from the container is that the truck raises the tip up and the chips begin to slide onto the screw conveyors to the rear of the container. Screw conveyors transport the chips to the blower's turbine, which produces the force for pneumatic blowing. The turbine is powered by a hydraulic motor installed in the blower, and the motor is connected to an oil tank with hydraulic hoses.

\subsection{Alternatives for the Blowing Supply Chain}

The blowing concept requires a great deal from the entire supply chain to make it work, from the sourcing of the raw material all the way to the end users. The starting point of the concept is that the supply chain needs high-quality wood fuel from regional fuel suppliers. The blowing concept can be integrated into two alternative supply chains for wood chips, either a terminal chipping chain or a roadside chipping chain (Figure 4). Thanks to integration, the blowing concept creates a new supply chain for wood chip delivery, which will be called the blowing supply chain.

This chain starts with the purchase of a pruned trunk from the roadside. Depending on the chipping chain, the trunks are chipped either by the roadside directly into the blowing truck or at the terminal to the storage stack after log transport. The terminal chipping chain also entails another extra work step, loading with a wheel loader, when compared to a direct roadside chipping chain. The wood chips are then transported to the destination, where they are blown 
with a hose into the customer's fuel storage system. Wood chip sites may include district heating plants, heat containers, or larger-sized properties. The last two sites are usually located in a difficult location, making unloading impossible with a normal chip truck. The new mobile heat containers that have entered the market are suitable for the heating of large-sized industrial buildings as well as larger-sized family houses that need a new heating solution to replace an expired oil boiler.

\section{Material and Methods}

The blowing tests were carried out at a typical district heating plant, in the region of central Finland (Figure 5). The tests were conducted both in summer and in winter to assess the success rate of chip feeding into a blower even in freezing temperatures. The tests took place between autumn of 2019 and winter of 2020. In total, five blowing tests were performed. In each individual test, one full container of wood chips was pneumatically unloaded. The outdoor temperature was higher than $4^{\circ} \mathrm{C}$ in tests 1,2 , and 5, while in tests 3 and 4 the temperature was cold enough that frost persisted. The outdoor temperature was $-15^{\circ} \mathrm{C}$ in test 3 and $-7^{\circ} \mathrm{C}$ in test 4 .

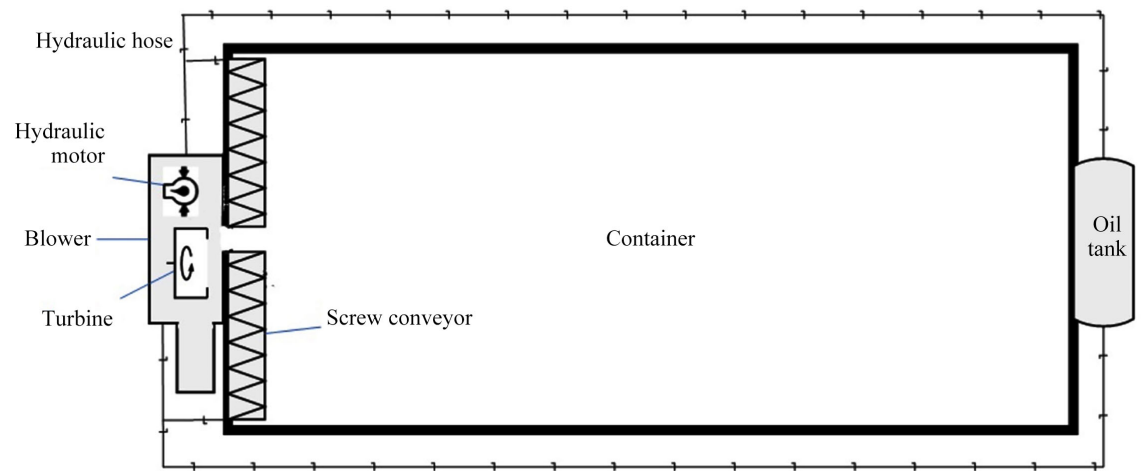

Figure 3. Diagram of the chip blower in the transfer container.

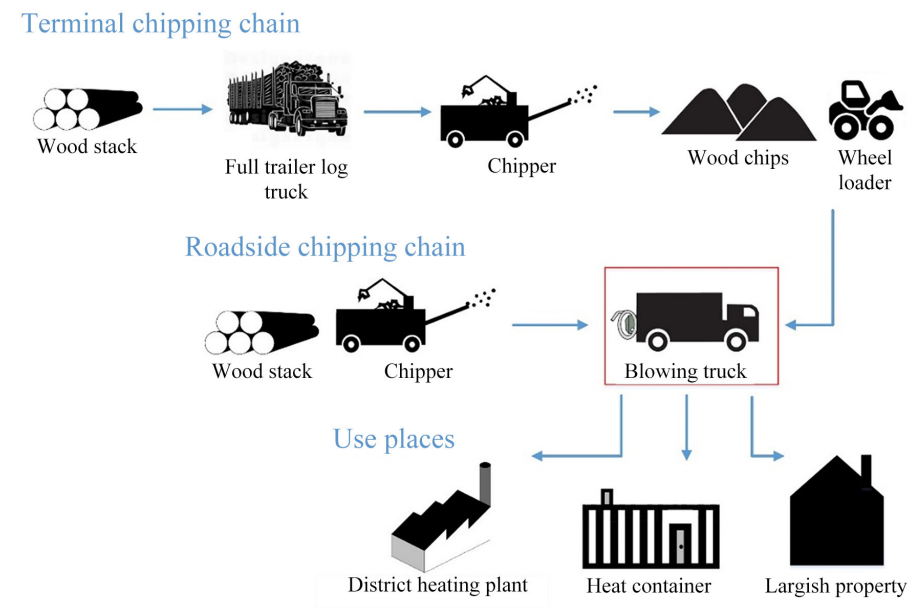

Figure 4. Diagram of the integration of the blowing concept into the most common biomass chipping chains. 


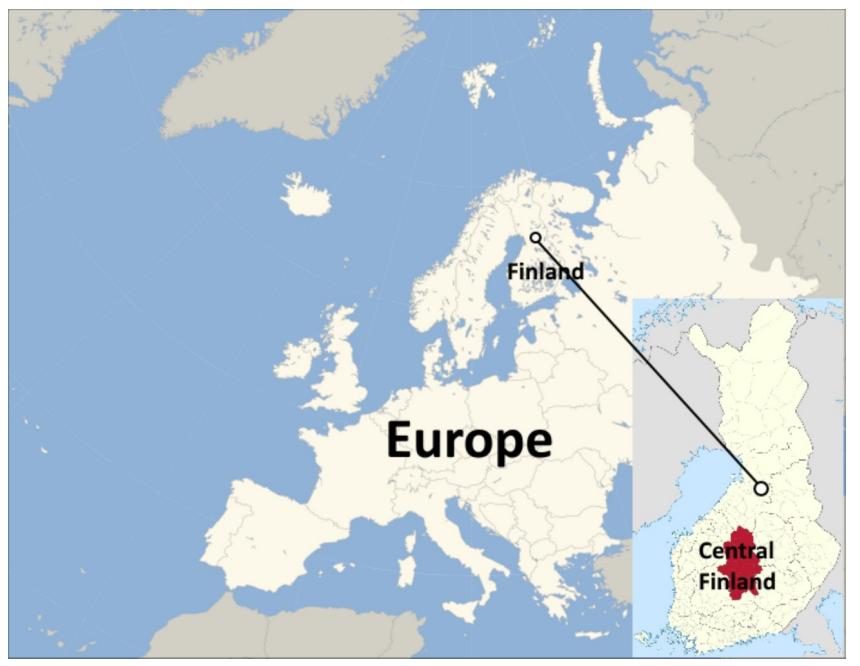

Figure 5. Central Finland on a map of Europe.

The aim was to arrange the blowing tests in connection with typical wood chip deliveries to the district heating plant, thus eliminating the need for separate deliveries. The truck was the same vehicle that had transported wood chips to the heating plant before, but it used the KOME composite transfer container installed with the chip blower (JYJ600). It was assumed that the revolution speed and power of the truck's engine or hydraulic motor would not increase as the chip load increased during the tests. In this case, each test tended to run through a specific revolution range.

The maximum pressure of the hydraulic system of the chip blower was limited to 350 bar by a valve. According to the blower manufacturer Romanet, at a pressure of 250 bar the optimal rotation speed of the blower turbine should be achieved, which is 4500 RPM [8]. This is where the main point of this study comes in. The maximum pressure of the truck's hydraulic system used in the tests was 160 bar, which was checked via measurements before starting the actual blowing tests. In this case, the pressure of the truck's hydraulic system was the single greatest limiting factor when considering the peak revolutions of the turbine.

The following section describes in more detail the feasibility of each measurement method and the properties of the wood chips used in the blowing tests.

\subsection{Material}

During the tests, a sample of chips was collected from each container to determine the particle size distribution and another sample was collected to determine the moisture content. The chips were collected in the sample bags from several different sampling points according to the sampling standard SFS-EN ISO 18135:2017 [10]. All the sample bags were stored in a cold room at $5^{\circ} \mathrm{C}$ before the laboratory tests. The chip samples were analyzed according to the following standards for solid biofuels: SFS-EN ISO 14780:2017 (sample preparation), SFS-EN ISO 18134-2:2017 (moisture content), and SFS-EN ISO 17827-1 
(particle size distribution) [11] [12] [13]. For the latter standard, the specification differed from the standard for sieve sizes and shapes.

\subsection{Methods}

\subsubsection{Rotation Speed and Volume Flow}

The rotation speed of the turbine $(\mathrm{r} / \mathrm{min})$ was measured with a tachometer when blowing the chips. The test was performed by applying a piece of tape to the shaft of the turbine and measuring its rotation speed optically with a hand gauge. A normal-use CEM DT-6236B tachometer was used in the study [14]. The measurement accuracy of the device was $\pm 0.05 \%$, and the maximum measuring range was up to 100,000 RPM. Corresponding measurements were also made for an empty load, in which case the blower rotated without chip resistance.

The volume flow of chips $\left(\mathrm{m}^{3} / \mathrm{h}\right)$ from the hose (diameter $150 \mathrm{~mm}$ ) was calculated in relation to the volume supplied $\left(\mathrm{m}^{3}\right)$ and the time used. In the tests, the volume flow was calculated for the time period, which was used for blowing the entire container $\left(36.6 \mathrm{~m}^{3}\right)$. The running time was always stopped if any interruptions occurred. The length of the hose was kept constant, being two meters during the tests.

\subsubsection{Speed of Chip}

The speed of chip $(\mathrm{m} / \mathrm{s})$ was measured at the mouth of the hose when the motion of the individual chips was recorded with the help of a video camera. The measuring distance of the chips was one meter, and the distance was marked out with a standing wooden stick. The speed of chip was subsequently calculated using a slow-motion video image. Speed $v$ was calculated using the formula, $v=d / t$, where $d$ was the traveled distance and $t$ was the used time. Unfortunately, chip speed measurements were performed only for the last three blowing tests. In each blowing test, the speeds of six different chips were measured and the average speed for the chip calculated. In this case, it was also possible to calculate the standard deviation for the average speed.

\subsubsection{Noise Level}

Standard ISO 1680:2013 applies to noise measurements for the wood chip blower [15]. The standard is called the Acoustics-Test code for the measuring of airborne noise emitted by rotating electrical machines. This standard defines a less accurate test method to be used when the measurement conditions do not meet the requirements of an exact standard. The noise level of the blower (dB) was measured at several points at a constant distance from the blower (Figure 6). A normal-use Amprobe SM-10 sound meter was used in the study [16]. For the sound meter, the measuring range was $30-130 \mathrm{~dB}$, while the A-weighting and its frequency range was $31.5-8000 \mathrm{~Hz}$. The standard recommends a measurement distance of one meter, but measurement distances of $5 \mathrm{~m}, 10 \mathrm{~m}, 15 \mathrm{~m}$, and $20 \mathrm{~m}$ were also used for the noise measurements. These alternatives gave some indication of the sound behavior in open ground. 


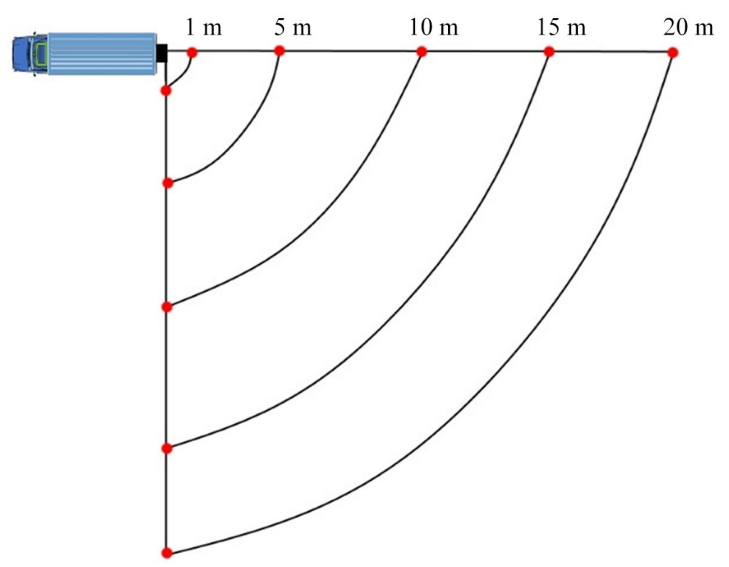

Figure 6. Principal view of the noise measurement points marked with red dots.

Although it is important to know the noise level of the machine under load, almost all current national standards define the machine's measurement mode when idling and define possible noise limits related to it. The reason for this has to do with measurement difficulties. In the blowing tests, noise measurements were performed with the blower in two different cases: in the first case, the hatch of the protective box was closed, while in the second case the hatch was open at a 180-degree angle. According to the safety instructions for the blower, the hatch of the blower must be closed and locked during operation, but the hatch was kept open only for measurement purposes at that moment. The measuring height was $1.5 \mathrm{~m}$ at each measurement. The A-weighting was used in all blowing tests.

\section{Results}

\subsection{Quality of the Wood Chips}

The quality results for the wood chips are presented in Table 3. The average moisture content of all the chips was $35.4 \mathrm{w}-\%$, and we detected no large deviations in the results for the individual moisture samples. Only the chips of the fifth test were clearly drier than the others, being at $28.3 \mathrm{w}-\%$. We also detected no large deviations in the particle size distribution of all the chips. However, the distribution shows that the chips from the fifth test were $11 \mathrm{w}-\%$ higher than the average in the size class of $16-31.5 \mathrm{~mm}$. Correspondingly, in the size class $2-4$ $\mathrm{mm}$ the weight proportion was $6.8 \mathrm{w}-\%$ lower than the average.

\subsection{Rotation Speed and Volume Flow}

The rotation speed of the turbine and the dependent volume flow of the chips are shown in Figure 7. A clear relationship between the two can be seen in Figure 7. We drew a polynomial trendline on the graph to clarify the consistent similarity of the measuring results. During of all five blowing tests, we measured the free running zone of the blower for the area at 3119 - 3405 RPM. 
Table 3. Quality results for wood chips.

\begin{tabular}{ccccccccccccc}
\hline Tests & $\begin{array}{c}\text { Chip } \\
\text { material }\end{array}$ & $\begin{array}{c}\text { Moisture } \\
\text { content } \\
(\mathbf{w}-\%)\end{array}$ & \multicolumn{9}{c}{ Weight distribution according to the size class } \\
$(\mathbf{w}-\%)$
\end{tabular}

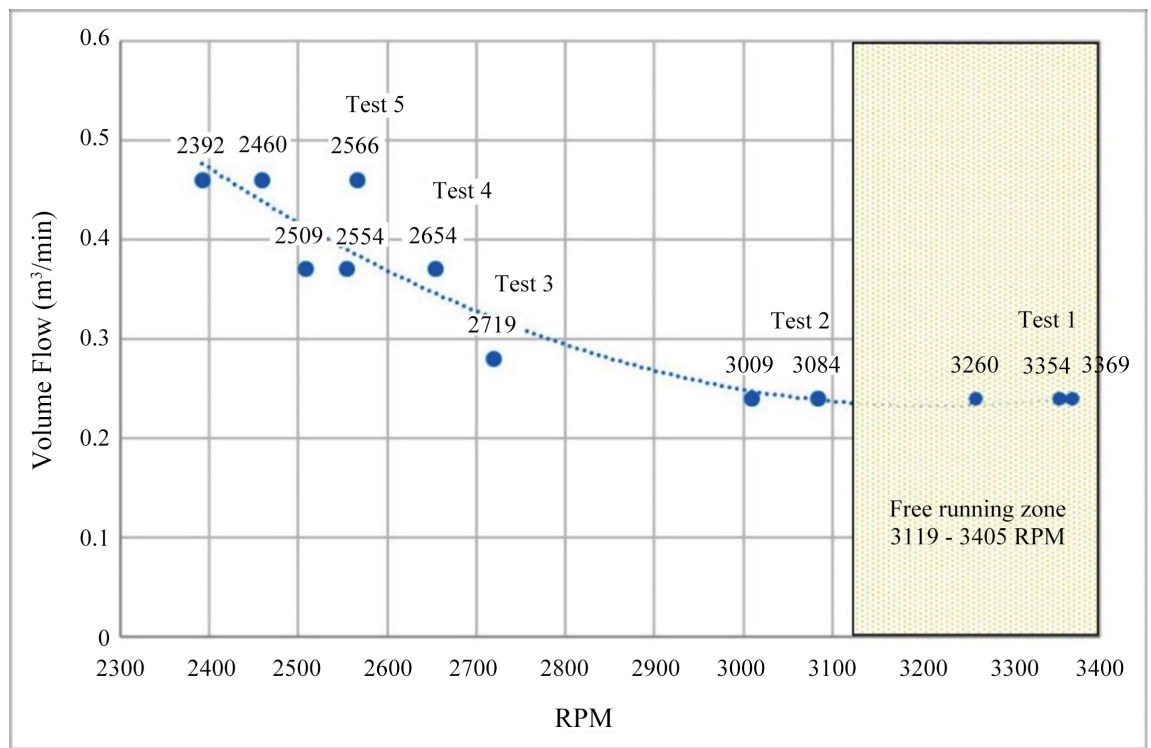

Figure 7. Rotation speed of the turbine affected the volume flow of chips.

\subsection{Speed of Chip}

We measured the speed of chip from the last three blowing tests. We defined the average speed for the chip in each blowing test (shown according to the prevailing RPM in Figure 8). The standard deviations of average speeds are also noted in the same figure. Figure 8 shows that when the RPM was low (being about 2400 RPM), the average speed of chip was clearly high (being $29.9 \mathrm{~m} / \mathrm{s}$ ).

\subsection{Noise Level}

We measured the noise level of the blower in each blowing test, and their average results are shown in Figure 9. Figure 9 shows that the noise level was higher in the vertical measurements to the blower. Respectively, the measurements taken from the side gave slightly lower noise levels. In addition, the protective box had muted the noise. For example, at a distance of one meter, the noise level was muted by approximately $1-7 \mathrm{~dB}$. 


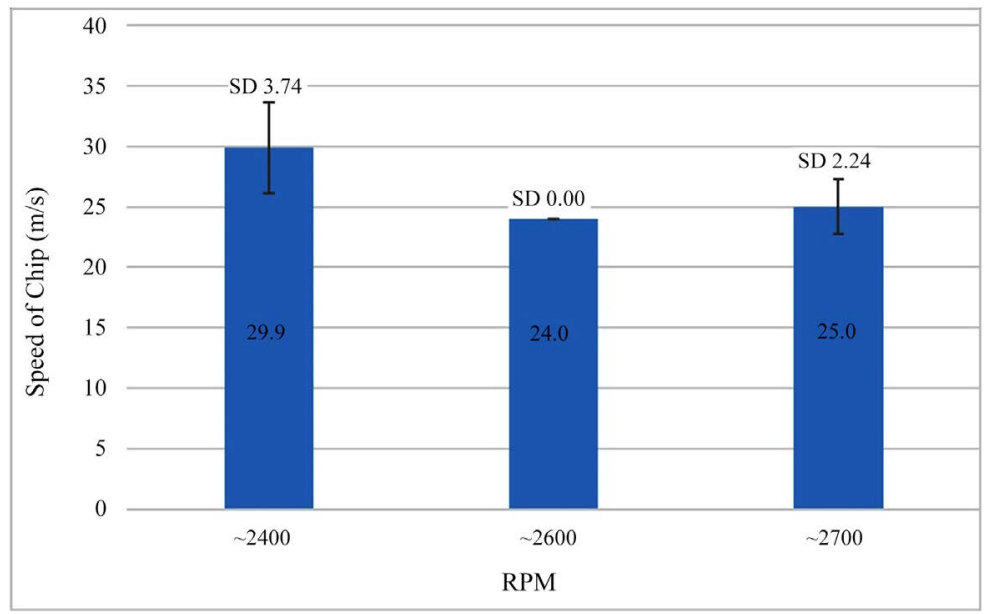

Figure 8. Average speed of chip according to the RPM (the standard deviations for speeds are also marked).

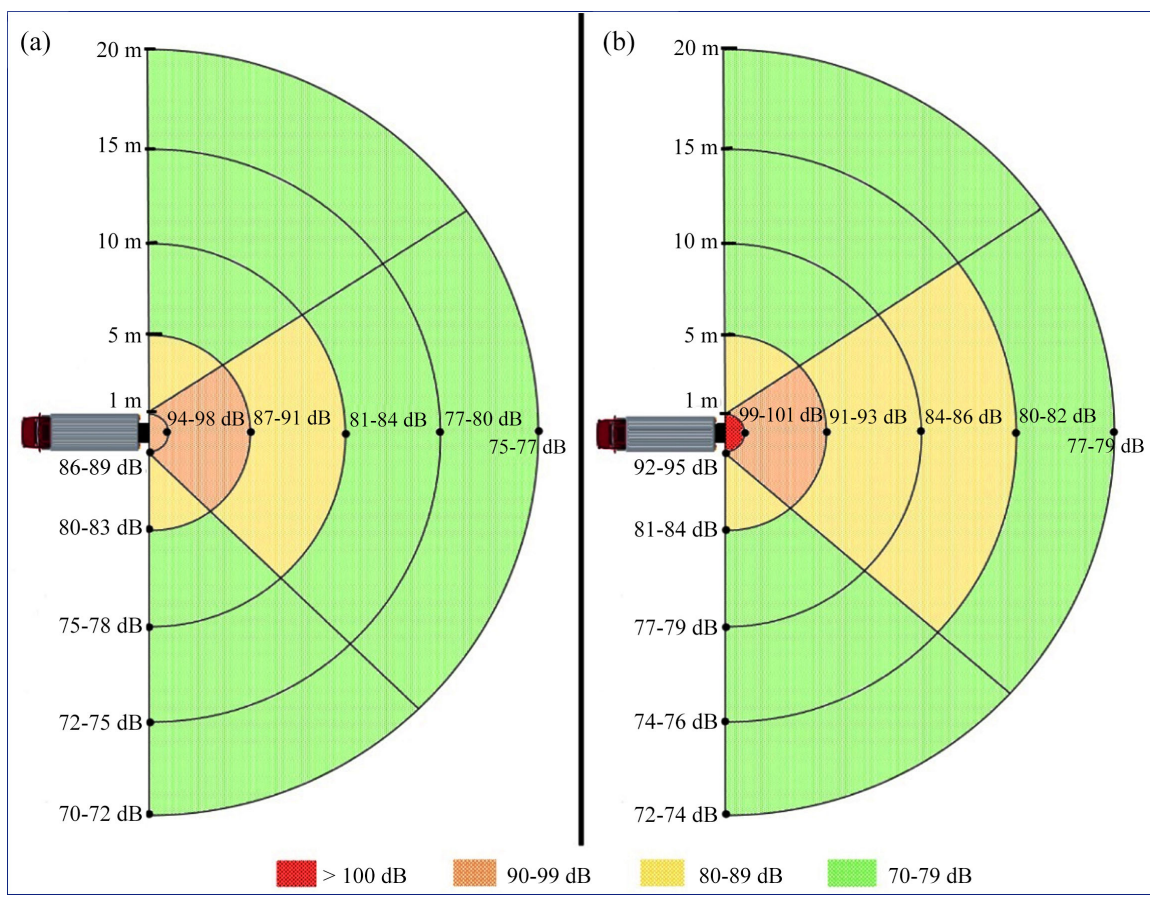

Figure 9. Results for blower's noise level measurements. In case (a), the hatch of the protective box was closed and in case (b), the hatch was open at a 180-degree angle.

\section{Discussion}

In this paper, we introduced a wood chip blowing system that can pneumatically blow wood chips straight from the container truck into a storage silo through a hose. This system can be exploited for hard-to-reach delivery places, such as narrow passes where rear unloading and side unloading are not possible. Thus, this system can be a new logistics solution for small chip deliveries. Normally, the final delivery locations for wood chips can be small district heating plants, large properties or, for example, mobile heat containers offering a new innovation in heating. This special heat container can also be a solution for reducing 
the use of fossil fuel.

The blowing system itself can be used for other materials in addition to wood chips. The system can also be used to blow the pellets, but at that time a slightly different type of blower must be used, the blades of which do not break the structure of the pellet. In addition, it can also be used in industry, on farms, for infrastructure and landscaping, for gardens, and for many other applications. Lastly, this system also eliminates the ground handling of biomass at the delivery destination when additional equipment is required, such as bucket tractors and wheel loaders.

No comparative study was found in the literature for this pneumatic wood chip transfer. In addition, no similar studies have been performed with a similar blowing device in the past, and information was found in the literature mainly on various old patents for totally different wood chip ejectors or separators. Therefore, no comparative study with other similar studies was performed in this paper.

The actual blowing tests yielded quite expected results. However, the tests did not achieve as much flow capacity for the JYJ600 model as the system developer, Romanet Sarl, has reported (Table 2). The tests did not attempt to achieve full capacities for the volume flow of the chips, as the purpose was only to measure reliable rotation speeds. During the tests, in several instances the blower was close to choking when too many wood chips moved from the feed screw to the blower. In this case, both were overloaded, and the oil pressure was not sufficient to maintain the turbine rotation speed, especially if long sticks clogged the blower's hose. Such situations can occur especially when delivering wet and sticky chips (length over $10 \mathrm{~cm}$ ). In these operating situations, the blower user hears a sound indicating that the feed screw's speed must be reduced. Therefore, it is recommended that only good-quality wood chips be used in the chip blower, since wood chips made with a crusher can contain long sticks.

For the whole blowing system, it should be remembered that both the screw conveyors and the blower take power from the hydraulic motor. In practice, if the blower's RPM is increased, then the rotation of the screw conveyors will slow down in proportion. For this reason, we can also observe from Figure 7 that if the volume flow capacity of the wood chips decreases, then the RPM of the blower increases. However, based on the results, it can be assumed that if we had further reduced the blower speed below 2400 RPM, then the volume flow of the wood chips would have increased even more significantly. Unfortunately, this trendline development is left to speculation, as we did not manage to do so during the test days.

During the tests, we also measured the flight speed of the chips from the end of the hose. The average chip speed varied from 24 to $29.9 \mathrm{~m} / \mathrm{s}$, depending on the RPM of the blower. However, the number of measurement shots was low, and no major conclusions can be drawn from the results. The average speed had a standard deviation of up to $3.74 \mathrm{~m} / \mathrm{s}$, which is an indication of the varying speed at the end of the hose. The variability in average speed was certainly 
caused by the uneven feed of the chips into the blower, with the screw conveyors conveying the chips to the blower. In addition, the irregular slipping of chips to the screw conveyors caused an imbalance for the power of the hydraulic motor. A few times the truck had to be moved or the tipper lifted so that the chips would start to slide freely onto the screw conveyors.

The last thing to be measured was the noise level of the blower, and it was measured in each test. According to the average test results, hearing protection is always required near the blower when the system is running. When the hatch of the protective box was closed, the noise level at a distance of one meter in the vertical measurements was $94-98 \mathrm{~dB}$. Correspondingly, when the hatch was open the noise level was as high as $99-101 \mathrm{~dB}$. Less than $80 \mathrm{~dB}$ was reached after a distance of ten meters with the closed hatch and after a distance of 15 meters with the open hatch. Correspondingly, noise measurements from the side reached less than $80 \mathrm{~dB}$ after only five meters in both hatch cases.

In the future, research into this new blowing concept will continue with even more extensive analysis and comparative research with respect to other alternative fuel use concepts. This gives readers a better understanding of the competitiveness of the blowing concept in the described operating environment. The aim of the new concept is to replace oil sites for which a conventional wood chip solution is not suitable and where customers are accustomed to easy use, such as oil heating. The business concept could be a leasing model in which, for example, a mobile heat container is brought to the site for heat supply and taken away when the need runs out. Usually, though, customers are not ready for an expensive investment when the future is uncertain.

\section{Conclusions}

Normally, the range of the hydraulic oil pressure is selected when purchasing a truck according to its intended use. This study was limited to the truck's hydraulic system, but it showed that the entire blowing system works in practice. Measured in terms of blowing capacity, performance was not quite optimal for this particular truck, which had limited oil pressure. This means that if future studies aim to assess a turbine's rotation speed of more than 4500 RPM, then the truck's hydraulic motor would have to be replaced with a more efficient one and, at the same time, an oil cooler would need to be added to the truck. With these changes, a future study would be able to achieve the blowing capacities declared by the manufacturer.

This new overall solution offers many regional benefits because it can reduce dependence on imported oil in heating. The heat can be produced from local biomass, and it is possible to achieve emission benefits with climate-neutral fuel. In addition, it is possible to improve the business opportunities of the companies in the region, whereby money from the supply chain remains in the local area for other uses. Chip heating also aims to save costs during the heating season in Nordic countries, as chips are normally cheaper than oil. 
The new blowing supply chain can reduce the use of fossil fuel at heating properties because the chain allows for hose transfer. The blowing phase of the truck load may be slower than the unloading of a normal truck from the rear by tipping. Nevertheless, this new blowing supply chain aims to tap into a new heating market in a way not possible before. The supply chain can reach new potential heating customers and can also respond to the challenge of the new era after oil heating.

\section{Acknowledgements}

The tests were carried out as part of the project Innovative Solution for Solid Biomass Delivery and Mobile Heat Containers to Replace Oil-fired Heating. The project was conducted from November 2018 to December 2020. It was primarily funded by the Regional Council of South Savo from the European Regional Development Fund. The project also received funding from the Energy Foundation of Suur-Savo.

\section{Conflicts of Interest}

The authors declare no conflicts of interest regarding the publication of this paper.

\section{References}

[1] IPCC (2007) Fourth Assessment Report. AR4 Climate Change 2007: Synthesis Report. Intergovernmental Panel of Climate Change.

[2] Ministry of Economic Affairs and Employment (2018) Legislative Proposals: Coal Ban in 2029, More Transport Biofuels and More Biofuel Oil for Heating and Machinery. Press Release 18.10.2018.

[3] Official Statistics of Finland (2018a) Energy Supply and Consumption. Statistics Finland, Helsinki.

[4] Kärhä, K. (2011) Industrial Supply Chains and Production Machinery of Forest Chips in Finland. Biomass and Bioenergy, 35, 3404-3413.

https://doi.org/10.1016/j.biombioe.2010.11.016

[5] Official Statistics of Finland (2018b) Energy Consumption in Households. Appendix Table 2. Energy Consumption in Households by Energy Source in 2018, GWh. Statistics Finland, Helsinki.

http://www.stat.fi/til/asen/2018/asen 2018 2019-11-21 tau 002 en.html

[6] Rentizelas, A.A., Tolis, A.J. and Tatsiopoulos, I.P. (2009) Logistics Issues of Biomass: The Storage Problem and the Multi-Biomass Supply Chain. Renewable and Sustainable Energy Reviews, 13, 887-894. https://doi.org/10.1016/j.rser.2008.01.003

[7] Lämpösi Oy (2020) Energy Consulting for BioEconomy. https://lamposi.fi/

[8] Romanet Sarl (2020) Blowing Systems and Blowing Trucks. http://www.romanet-sarl.com/

[9] Kome Oy (2020) Kome Composite. http://www.kome.fi/tuotteet/kome-composite/

[10] SFS-EN ISO 18135:2017: en (2017) Solid Biofuels. Sampling.

[11] SFS-EN ISO 14780:2017: en (2012) Solid Biofuels. Sample Preparation. 
[12] SFS-EN ISO 18134-2:2017: en (2017) Solid Biofuels. Determination of Moisture Content. Oven Dry Method. Part 2: Total moisture. Simplified Method.

[13] SFS-EN ISO 17827-1: en (2016) Solid Biofuels. Determination of Particle Size Distribution for Uncompressed Fuels. Part 1: Oscillating Screen Method Using Sieves with Apertures of 3,15 $\mathrm{mm}$ and above.

[14] CEM (2020) Digital Tachometers. DT-6234B/6236B. http://www.cem-instruments.com/en/Product/detail/id/1302

[15] ISO 1680:2013 (2013) Acoustics-Test Code for the Measurement of Airborne Noise Emitted by Rotating Electrical Machines.

[16] Amprobe (2020) Products. Amprobe SM-10 Sound Meter. https://www.amprobe.com/product/sm-10/ 